\title{
The ATLAS Trigger Performance and Evolution
}

Valeria Bartsch, University of Sussex, UK for the ATLAS collaboration 


\section{Overview over the ATLAS Trigger System}

3 Level Trigger System:

- Level 1

- hardware based

- coarse granularity

- mainly muon chambers and calorimeters

- Level 2 (part of High Level Trigger)

- software based

- full granularity in regions of interest

- all subdetectors

- Event Filter (part of High Level Trigger)

- offline-like algorithms

- events put into output streams based on physics content

$44 \mathrm{~m}$ 
2011:

\section{Data Taking Efficiency of Trigger System}

\begin{tabular}{|c|c|c|c|c|c|c|c|c|c|}
\hline \multicolumn{7}{|c|}{ High Level Trigger (HLT) } \\
\hline Muon & Calo & CTP & electron & photon & muon & tau & jet & b-jet & $\begin{array}{c}\text { missing } \\
E_{T}\end{array}$ \\
\hline 99.0 & 100 & 99.8 & 99.3 & 99.3 & 100 & 99.9 & 98.6 & 99.9 & 99.3 \\
\hline $\begin{array}{l}\text { Luminosity weighted relative relative fraction of good trigger data quality delivery during } 2011 \text { stable beams in pp collisions at } \\
\text { ls=7 TeV between 13 March and 30 October (in \%). }\end{array}$
\end{tabular}

- Trigger Signatures at L1: concerning the hardware components and the central trigger reprocessor (CTP)

- Trigger Signatures at the High Level Trigger: identifying physics objects corresponding to fundamental particles

- Minor issues due to gas leaks in RPC and one run in 2011 with an issue of the jet algorithm after a hardware recovery

- L1 and HLT rates are calculated independently

- Overall Data Quality very high in 2011 and 2012 


\section{Evolution of the luminosity}

- 2011: 7 TeV centre of mass energy proton-proton collisions - 2012: 8 TeV centre of mass energy collisions

- up to $7.710^{33} \mathrm{~cm}^{-2} \mathrm{~s}^{-1}$ instantaneous luminosity

- 50 ns bunch spacing

- up to 38 mean collisions per beam crossing (pile-up)

- $19.28 \mathrm{fb}^{-1}$ recorded luminosity

- November - December 2011: heavy ion $(\mathrm{Pb})$ collisions $\rightarrow$ almost linear increasing instantaneous luminosity of the LHC

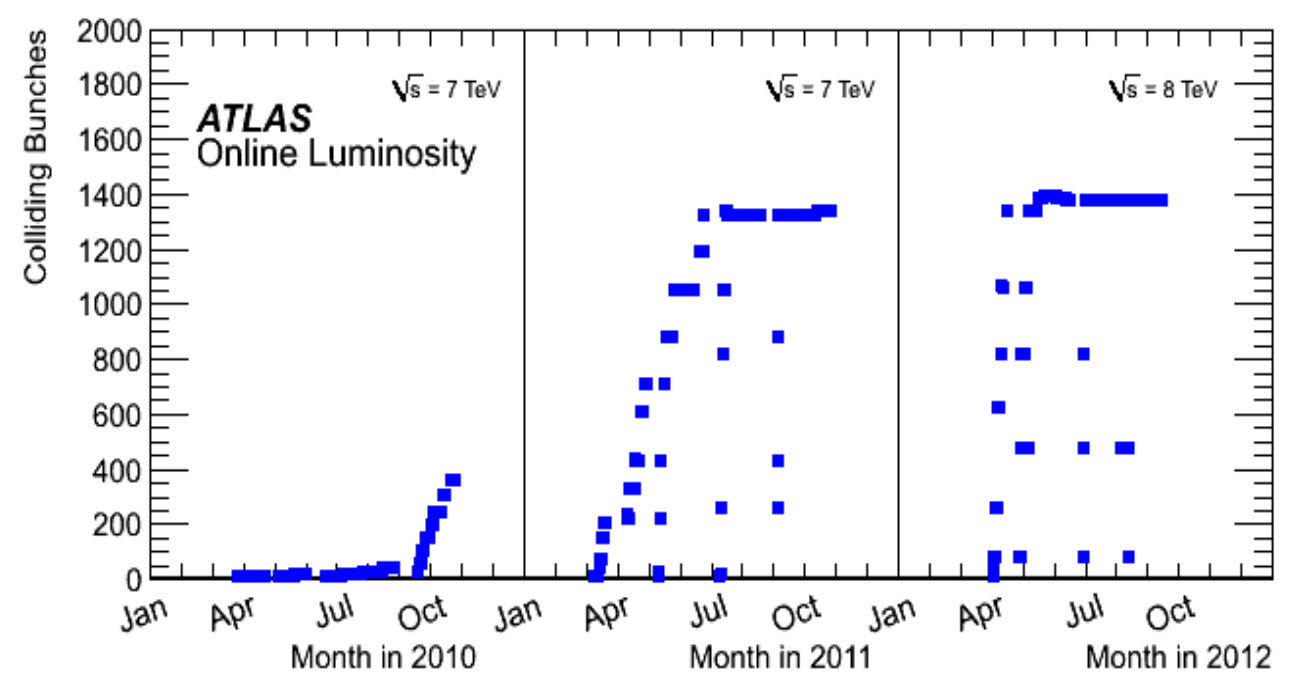

01 Nov 2012

V.Bartsch:

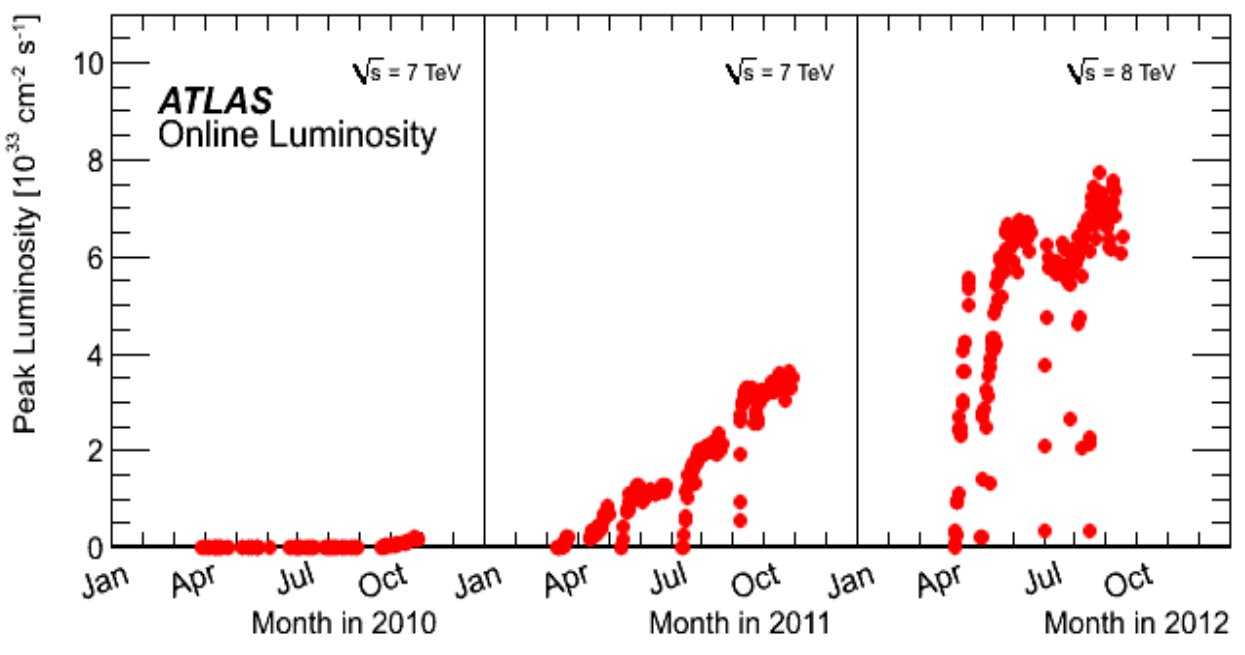




\section{Consequences on the Streaming}

- main output rates from Muons, Jet/Tau/Etmiss and Egamma streams

- Jet/Tau/Etmiss trigger rates decreasing during run

- increasing rate of data stored during 2012

- due to increasing rate of data streams introduction of delayed streams in 2012 (data will be analysed during shutdown periods)

- Average event size of raw data 1.2MB

- Total number of events: $210^{9}$ in 2012

- Total data stored for physics: about

$2 \mathrm{~Pb}$ in 2012

No. of

Data Streams in 2011 - 2012

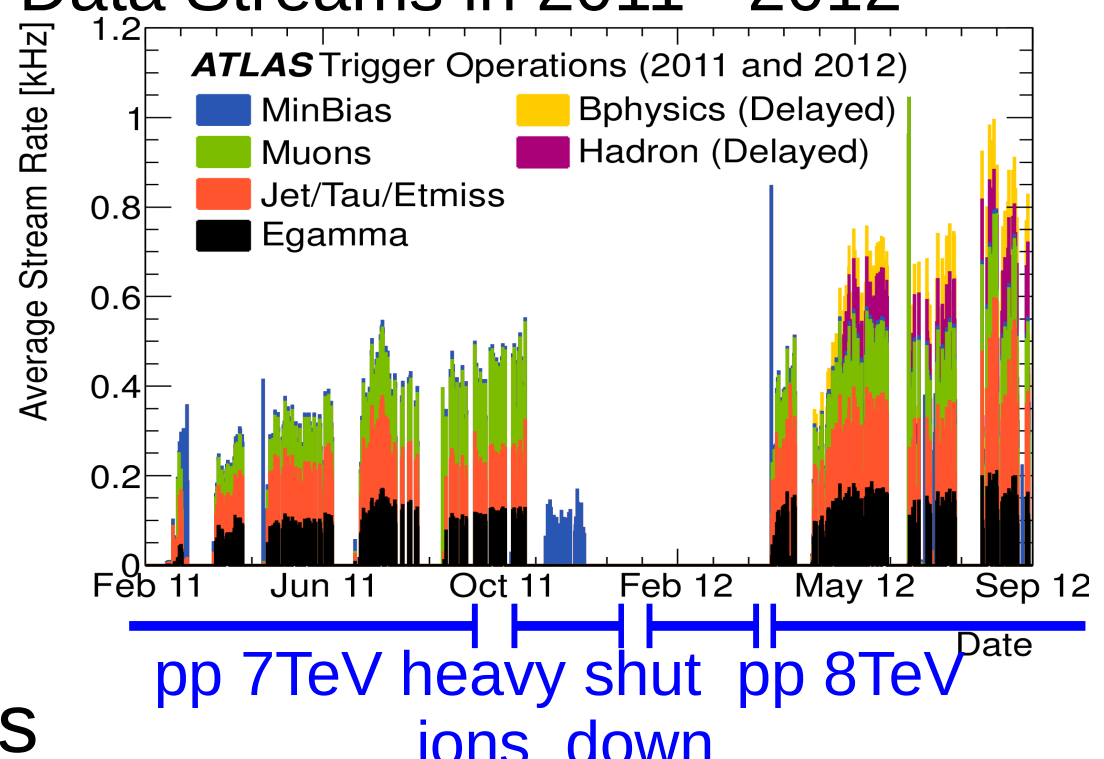

Data Streams in a typical run

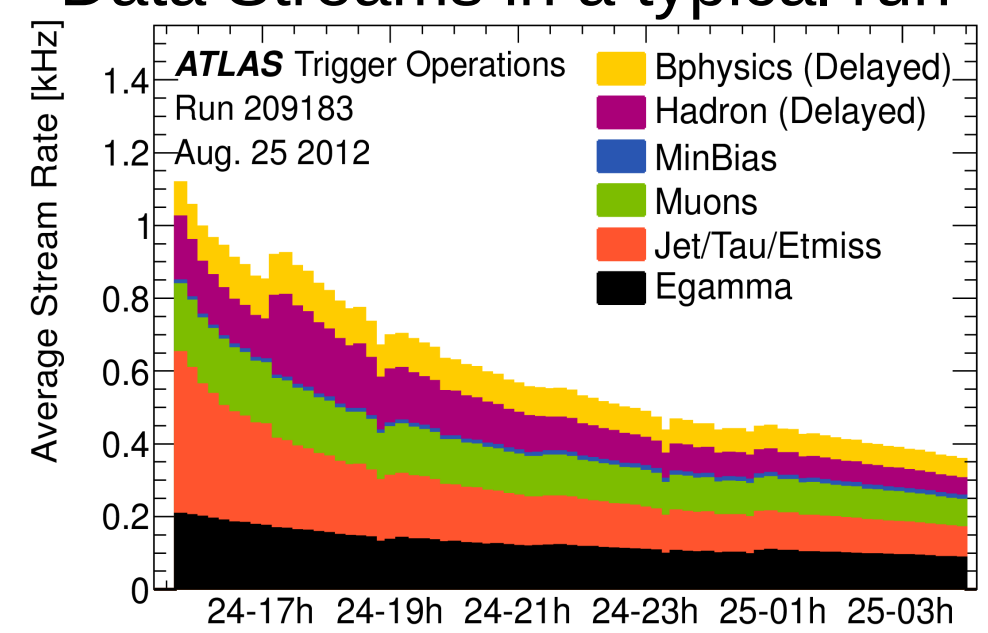

01 Nov 2012

V.Bartsch:

Interactions: $33 \quad 24 \quad 19 \quad 16 \quad 14 \quad 12$ cEs trithe 


\section{Trigger Menu for $7 \star 10^{33} \mathrm{~cm}^{-2} \mathrm{~s}^{-1}$}

- optimal distribution of bandwidth is critical

- driven by physics requirements and priorities

- most bandwidth given to most generic triggers

- frequent trigger changes complicate physics analyses

$\rightarrow$ in 2011 only 3 base menus for proton-proton collisions

- separate menu for heavy ion runs

\begin{tabular}{|l|l|l|l|}
\hline $\begin{array}{l}\text { Trigger } \\
\text { Signature }\end{array}$ & $\begin{array}{l}\text { Peak } \\
\text { L1 Rate }\end{array}$ & $\begin{array}{l}\text { Peak } \\
\text { L2 Rate }\end{array}$ & $\begin{array}{l}\text { Average } \\
\text { EF Rate }\end{array}$ \\
\hline Muon & $14 \mathrm{kHz}$ & $1200 \mathrm{~Hz}$ & $100 \mathrm{~Hz}$ \\
\hline $\begin{array}{l}\text { Electron / } \\
\text { Gamma }\end{array}$ & $30 \mathrm{kHz}$ & $2000 \mathrm{~Hz}$ & $140 \mathrm{~Hz}$ \\
\hline Tau & $24 \mathrm{kHz}$ & $800 \mathrm{~Hz}$ & $35 \mathrm{~Hz}$ \\
\hline Jets & $3 \mathrm{kHz}$ & $1000 \mathrm{~Hz}$ & $35 \mathrm{~Hz}$ \\
\hline $\begin{array}{l}\text { Missing } \\
\text { Energy }\end{array}$ & $4 \mathrm{kHz}$ & $800 \mathrm{~Hz}$ & $30 \mathrm{~Hz}$ \\
\hline $\begin{array}{l}\text { B-jets } \\
\text { B-physics }\end{array}$ & $5 \mathrm{kHz}$ & $900 \mathrm{kHz}$ & $45 \mathrm{~Hz}$ \\
\hline $\begin{array}{l}\text { Total } \\
\text { Total }\end{array}$ & $65 \mathrm{kHz}$ & $5500 \mathrm{~Hz}$ & $40 \mathrm{~Hz}$ \\
\hline
\end{tabular}

Group overlap accounted for in total 


\section{Consequences of luminosity increase on L1}

- L1 trigger rates mainly independent of pileup

- non-linear behaviour of missing transverse energy trigger items, multi-jet and forward jet trigger items

$\rightarrow$ use missing energy trigger items as an example to show improvements

L1MuX: L1 item of a muon candidate $p_{t}>X G e V$

L12MuX: L1 item of two muon candidates with a $p_{t}>X G e V$ L1EMX: L1 item of an electron/gamma candidate with E>XGel L1EMXVH: the same with high pileup optimisation

L1TAUX: L1 item of tau candidate with $E>X G e V$

L1_(F)JX: L1 item of a jet candidate with E>XGeV

L1_XEX: Missing Energy Item of missing energy of E>X

01 Nov 2012

V.Bartsch:

ATLAS Trigger Performa

Muon based Trigger Items

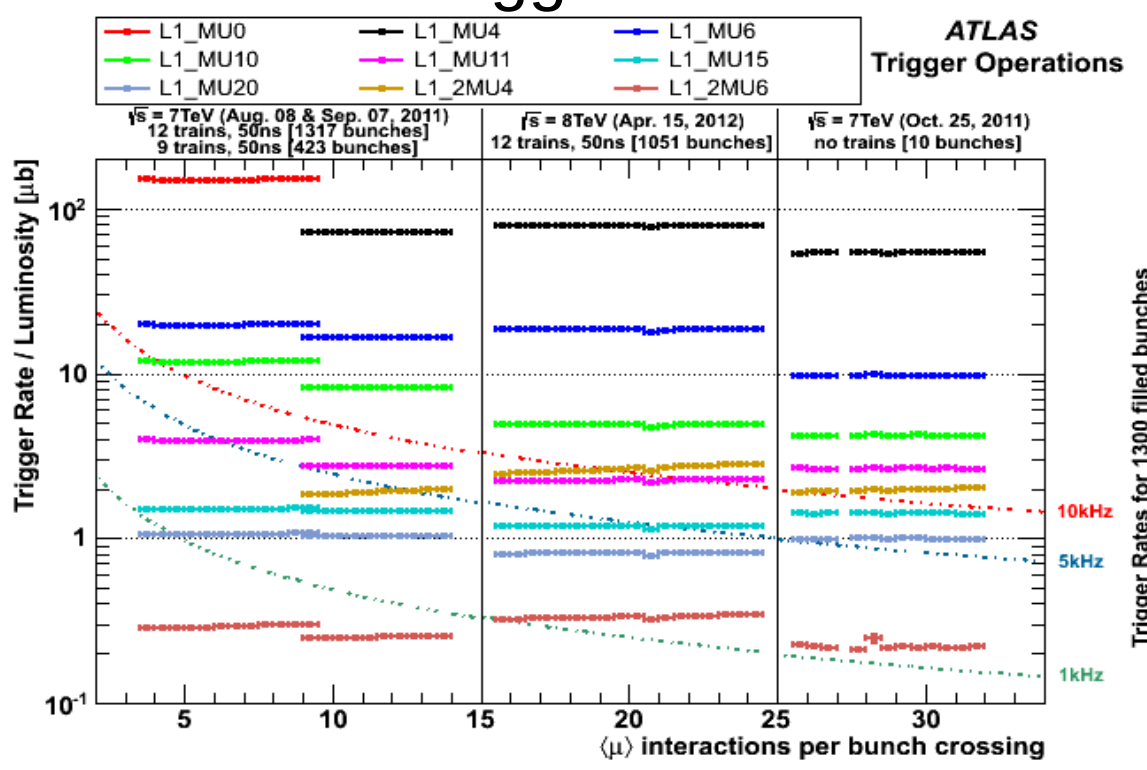

Calorimeter based Trigger Items

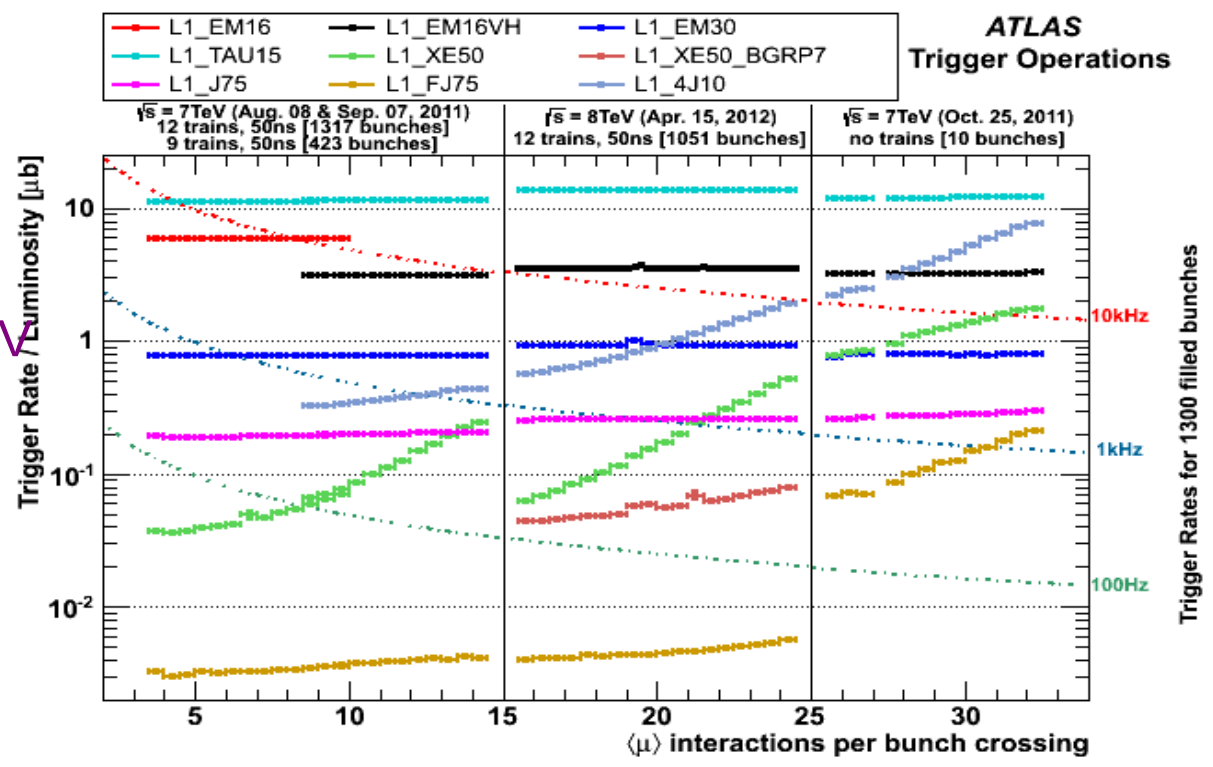




\section{Missing Energy Triggers: advances in L1}

bin 1: $\eta=3.1-3.2$

bin $2: \eta=3.2-3.5$

bin $3: \eta=3.5-4.2$

bin $4: \eta=4.2-4.9$

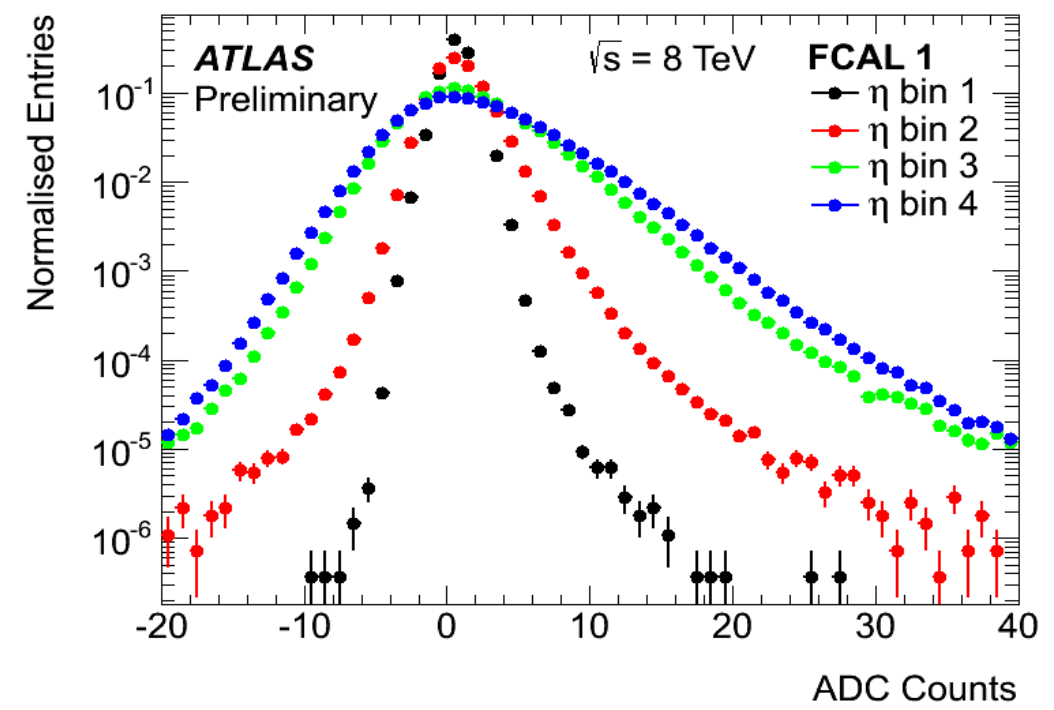

- out-of-time pileup noise from FCAL dominated Missing Energy Trigger rate

$\rightarrow$ raised noise cuts in FCAL region to suppress rate

$\rightarrow$ minimal impact on trigger efficiencies

$\rightarrow$ L1 thresholds lowered in 2012

01 Nov 2012

V.Bartsch:

ATLAS Trigger Performance
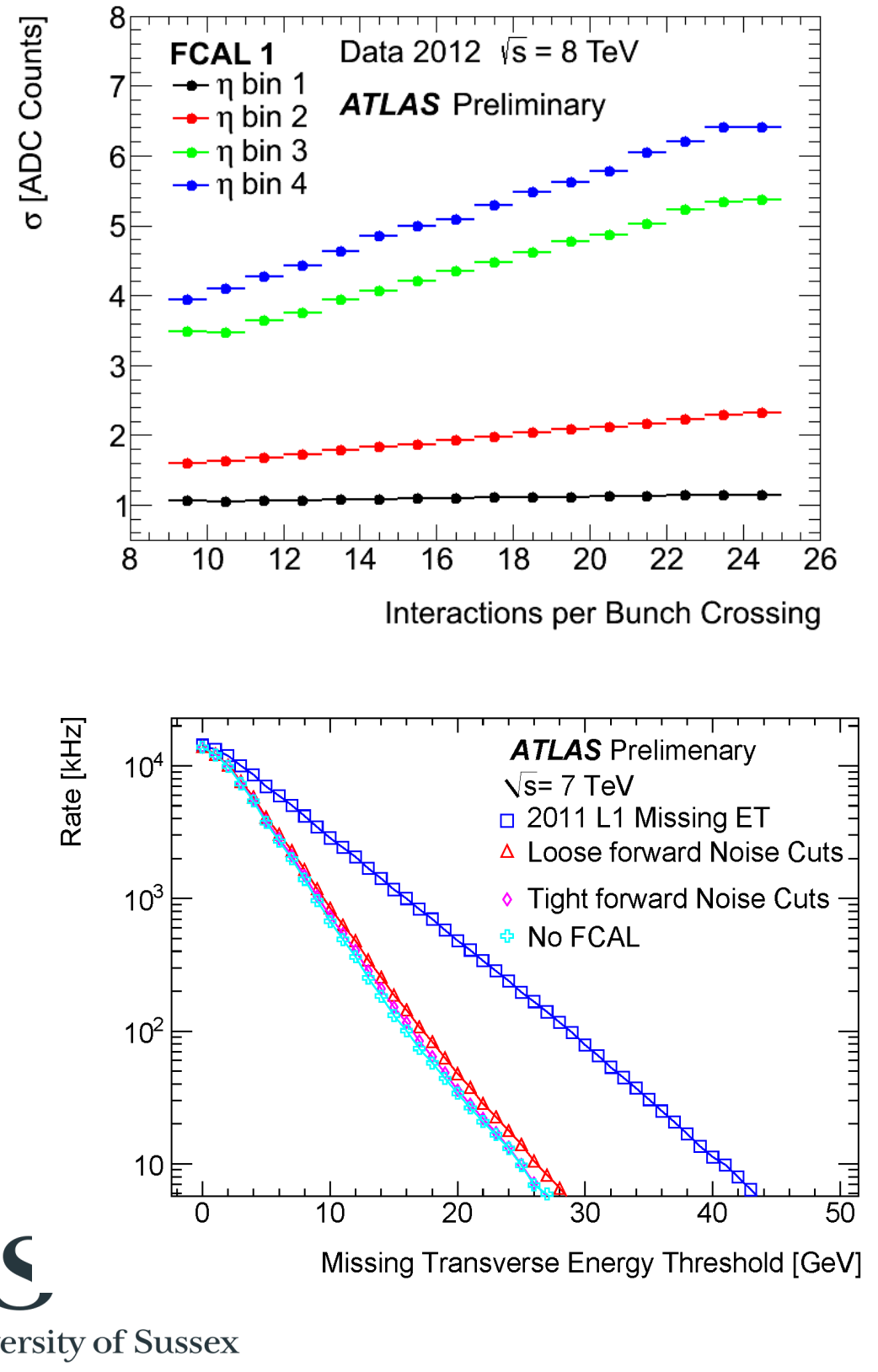


\section{Missing Energy Triggers: Out-of-Time Pileup}

Out of time pileup issues:

- Calorimeter pulses last longer than the time between bunch crossings and go to negative values

- Unbalanced overlaying of signal shapes from neighbouring bunches causes spike in L1 Missing Energy trigger compared to muon and electron/gamma trigger

$\rightarrow$ need to enable the Missing Energy Triggers only after this balance is reached

Trigger Rates for Missing Energy

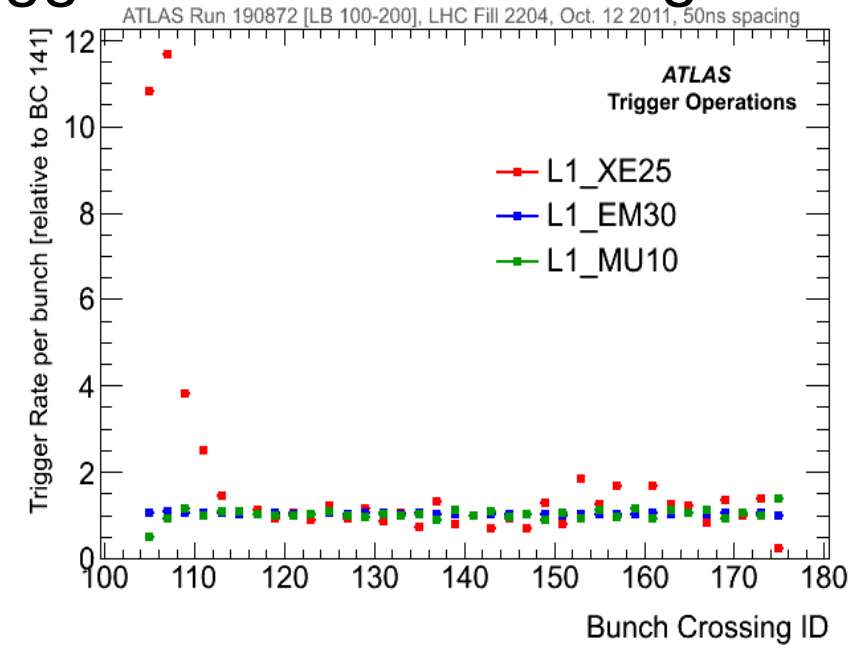

Schematics of a LAr signal pulse

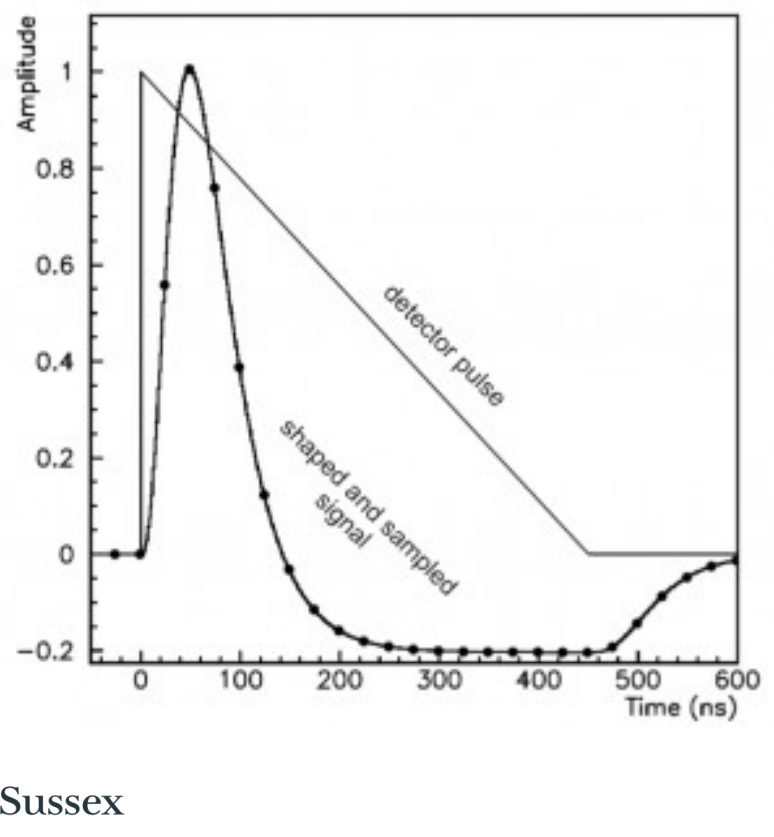




\section{Missing Energy Trigger: advances in HLT}

Advances in L2:

Resolution of Ex for 2 different algorithms

- In 2012: access to summed cell information rather than coarse L1 trigger towers

$\rightarrow$ resolution improves by about $50 \%$

$\rightarrow$ rejection rate improved by a factor $\sim 5$

$\rightarrow$ trigger thresholds lowered

Advances in EF:

- In 2012: cluster based MET / hadronic calibration (shared with Jet triggers)

$\rightarrow 40 \%$ resolution improvement

$\rightarrow$ allows to lower the trigger plateau value for the same threshold by about $30 \%$
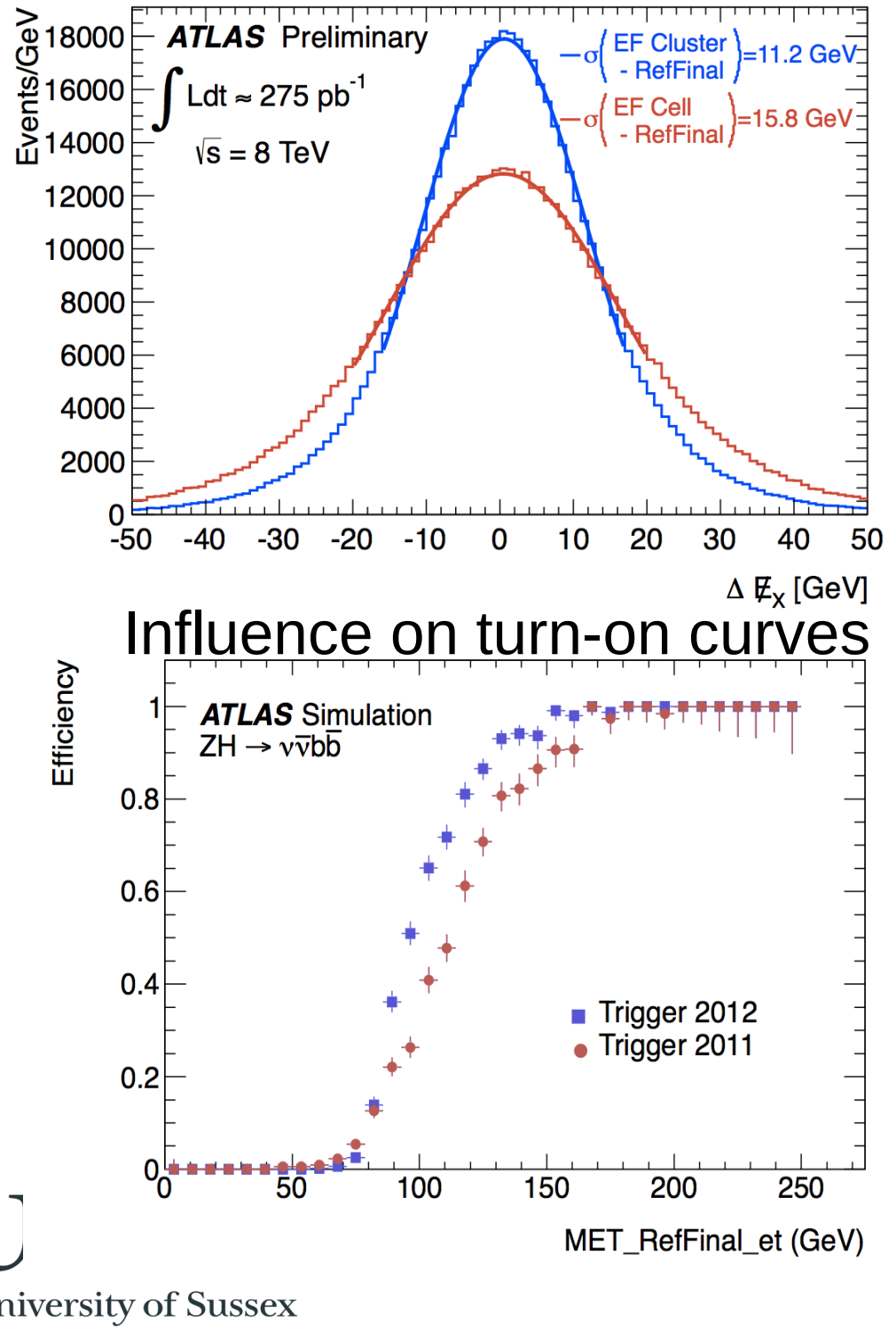


\section{Tau Trigger}

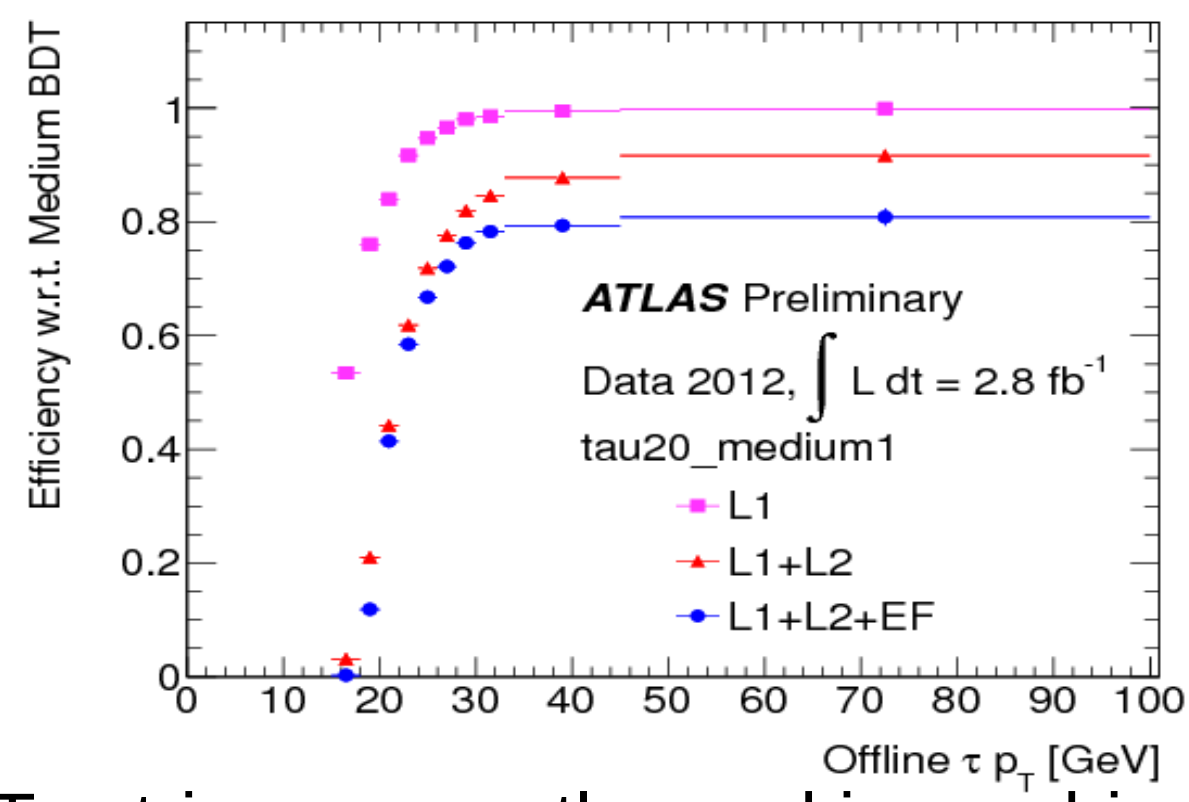

- Tau triggers mostly used in combination with $2^{\text {nd }}$ tau (hadronic or leptonic) or Missing Energy trigger

Significant improvement for 2012:

- much improved pileup robustness due to smaller cone sizes and vertex track cuts

- Event Filter uses multi-variate selection to increase rejection power significantly

01 Nov 2012
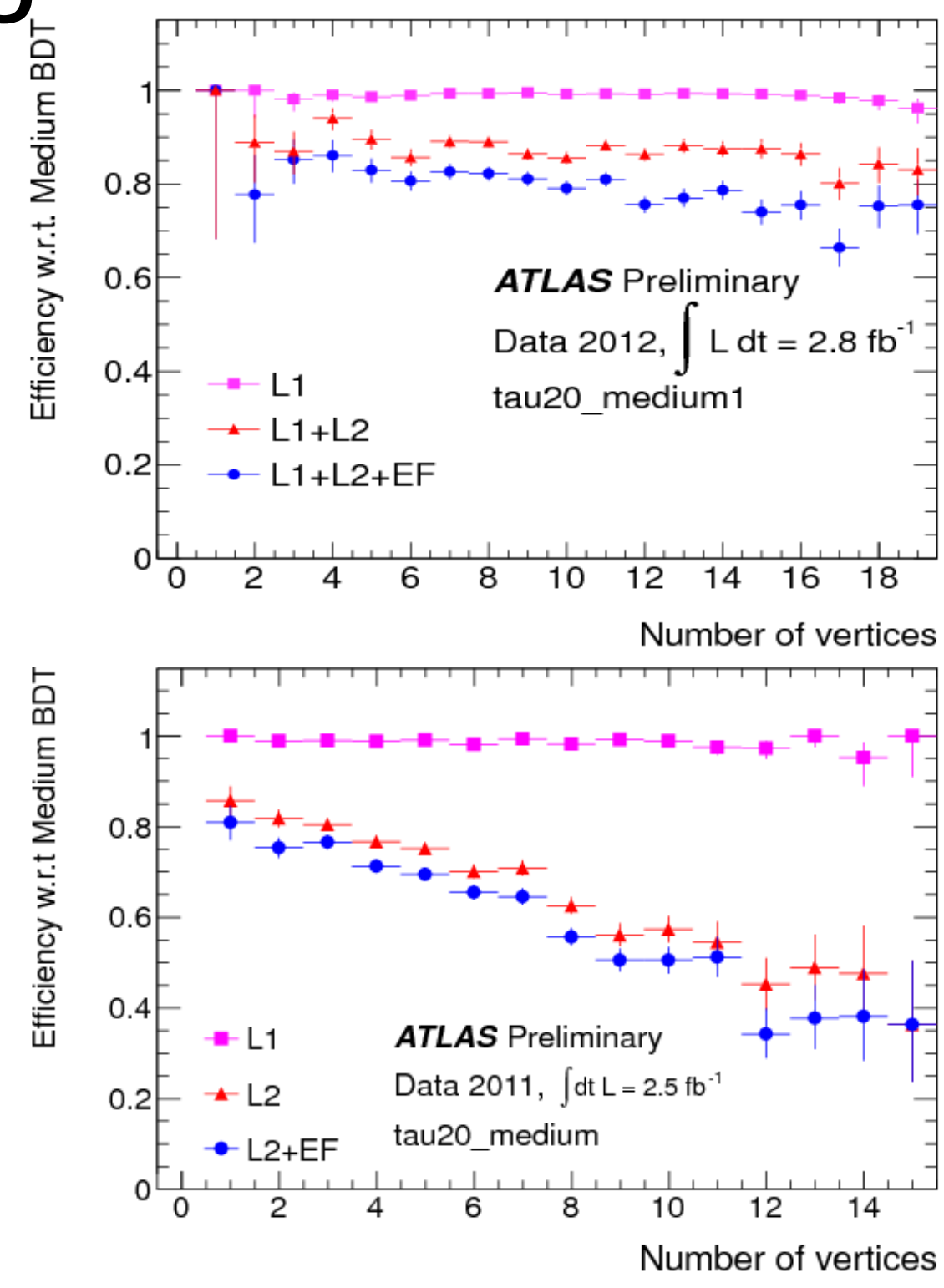

US
V.Bartsch:

ATLAS Trigger Performance 


\section{Physics with the ATLAS trigger}

- Tau trigger enables high acceptance of tau events

- Jet trigger alone would not be sufficient due to high thresholds

- Next step: look at branching ratios and spin to confirm standard model Higgs

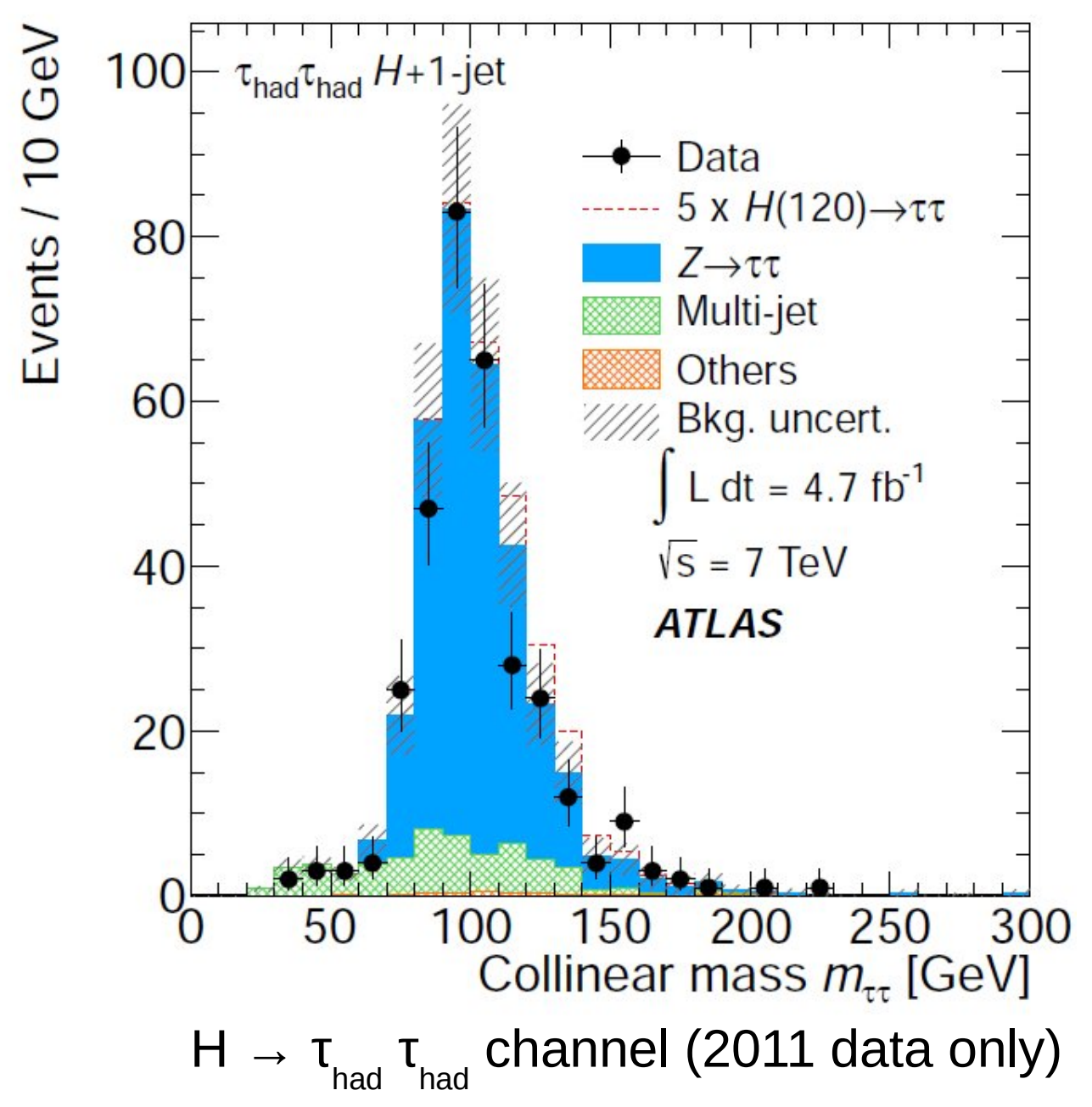




\section{Conclusions}

- ATLAS trigger operating successfully in 2011-2012

- Handling challenge of luminosity increases of LHC

- Significant improvements deployed for 2012:

- Shown at the example of the missing energy signature and tau triggers

- Retuned selection for high pileup conditions

- More advance HLT selection algorithms

- Trigger thresholds only raised minimally w.r.t. 2011 


\section{Backup slides}




\section{Muon Triggers}

2011:

- Muon trigger at pt>18 GeV/c

- Tightened L1 trigger due to out-of-time hits with 50ns beam spacing

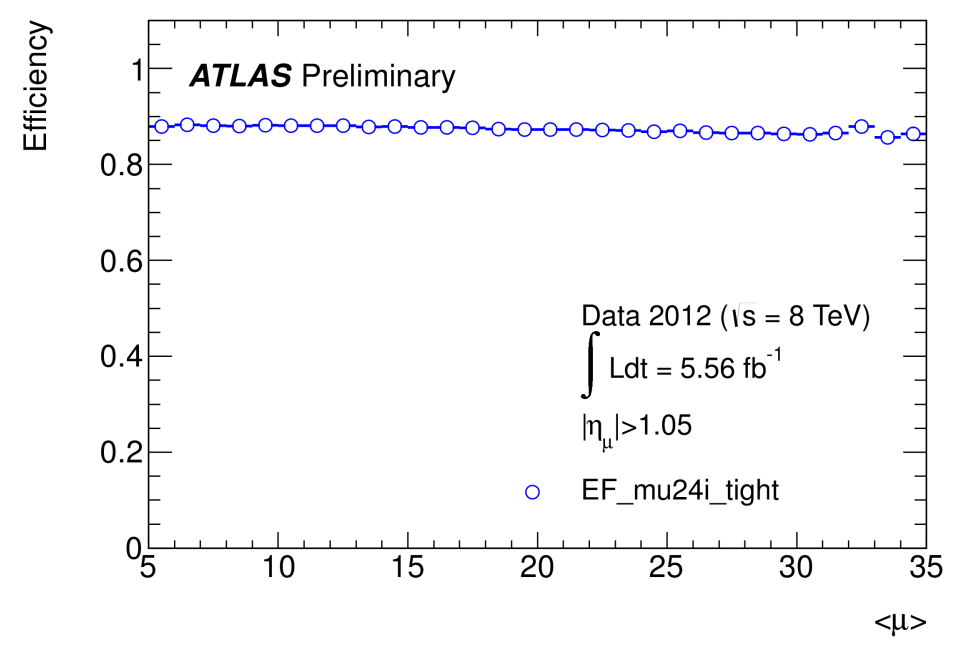

2012:

- Additional shieliding installed

- Raise to $\mathrm{pt}>24 \mathrm{GeV} / \mathrm{c}$

- Track isolation required (pileup robust)

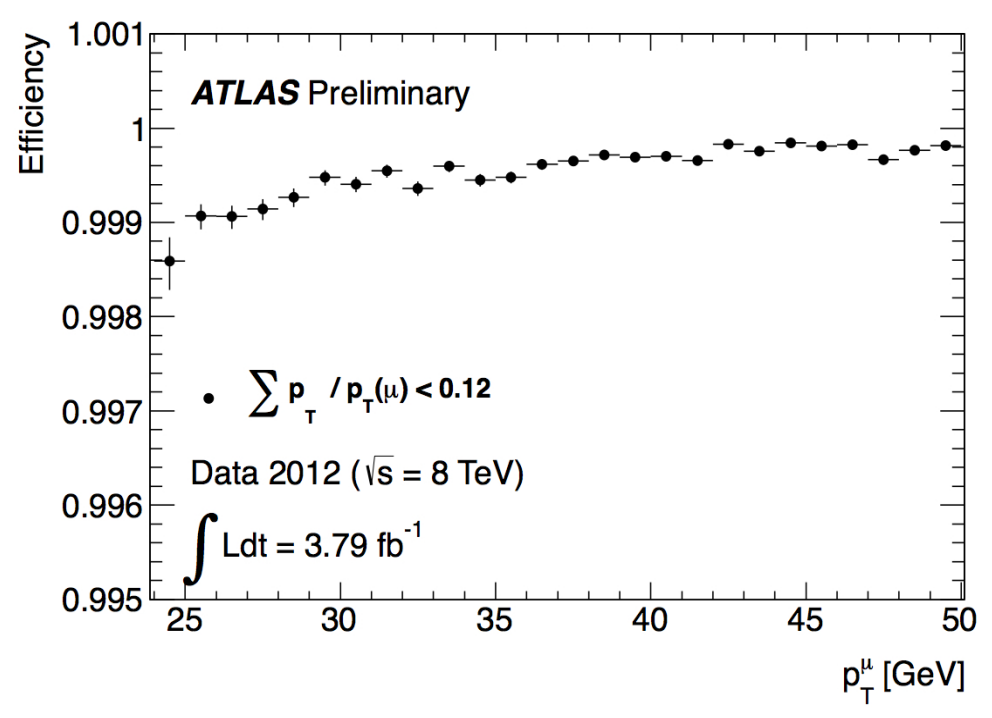




\section{Electron / Gamma triggers}

Electrons

- $25 \mathrm{GeV}$ and $2 \times 15 \mathrm{GeV}$ electron triggers

2011:

- Hadronic veto at L1

- Retuned HLT and offline electron ID

2012:

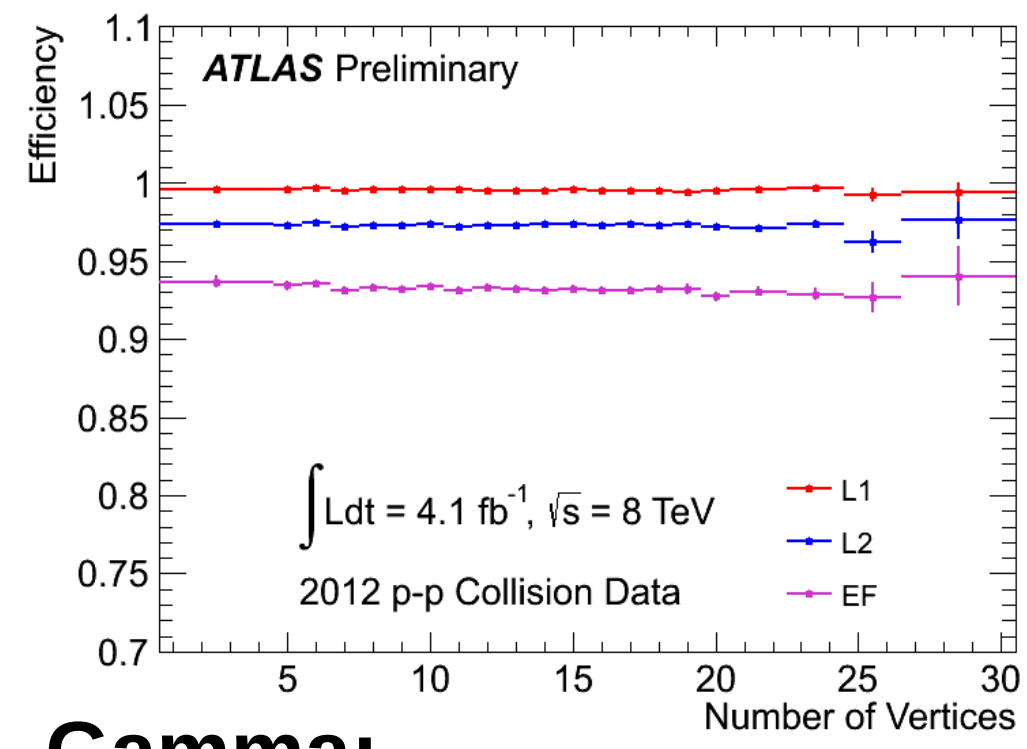

Gamma:

- Loosened pileup sensistive selection, but raised pt thresholds

- Raised L1 threshold

- Retuned electron ID for high pileup

- Track isolation required

- 2x20GeV trigger with somewhat tighter photon identification

- Added 3-photon trigger 


\section{Jet Triggers}

2012:

-Evolution away from Rol based triggers -Full scan reco of L1 towers for anti-kt jets

-Hadronic scale for HLT jets

-Noise threshold adjusted for higher pileup

-More advanced b-tagger (multi-variate, multi-jet vertex)

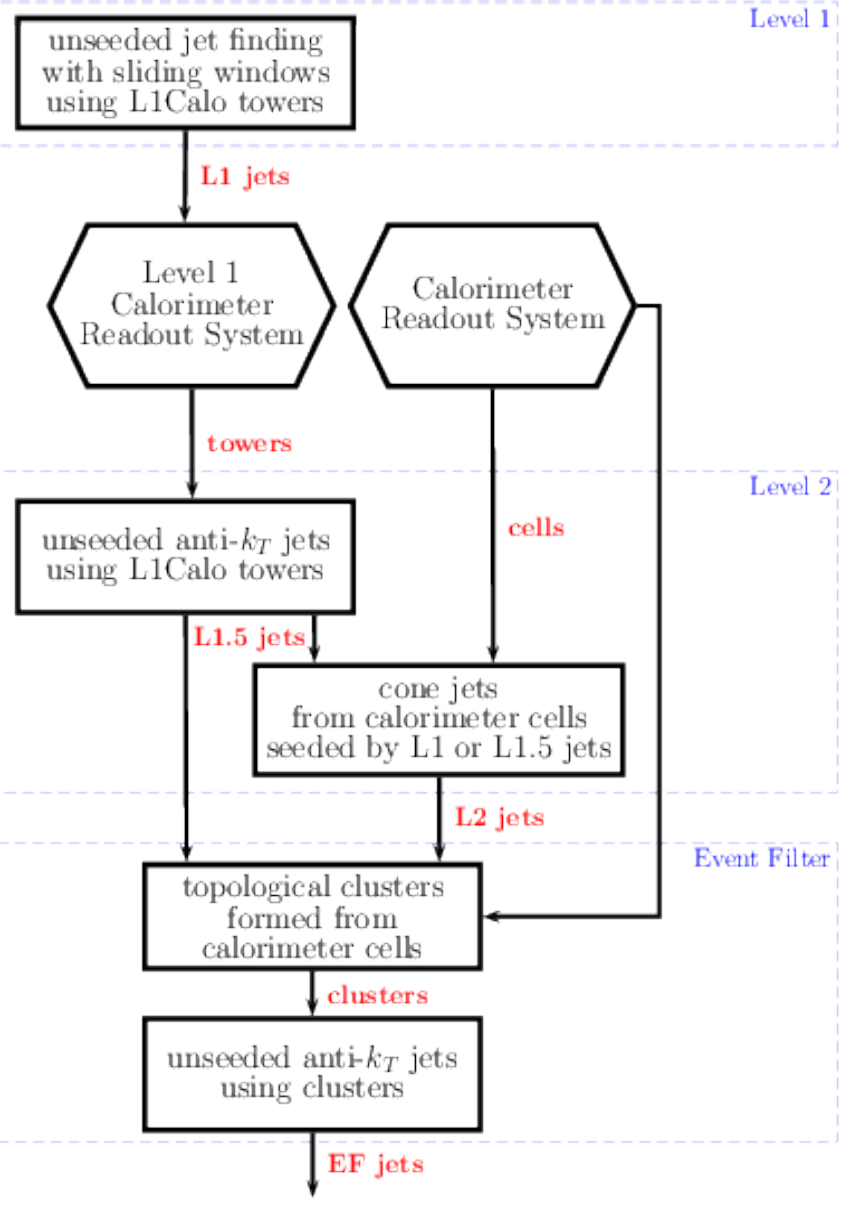

